\title{
KÄHLER-EINSTEIN METRICS EMERGING FROM FREE FERMIONS AND STATISTICAL MECHANICS
}

\author{
ROBERT J. BERMAN
}

\begin{abstract}
We propose a statistical mechanical derivation of Kähler-Einstein metrics, i.e. solutions to Einstein's vacuum field equations in Euclidean signature (with a cosmological constant) on a compact Kähler manifold $X$. The microscopic theory is given by a canonical free fermion gas on $X$ whose oneparticle states are pluricanonical holomorphic sections on $X$ (coinciding with higher spin states in the case of a Riemann surface) defined in background free manner. A heuristic, but hopefully physically illuminating, argument for the convergence in the thermodynamical (large $N$ ) limit is given, based on a recent mathematically rigorous result about exponentially small fluctuations of Slater determinants. Relations to higher-dimensional effective bosonization, the YauTian-Donaldson program in Kähler geometry and quantum gravity are explored. The precise mathematical details will be investigated elsewhere.
\end{abstract}

\section{INTRODUCTION}

The basic laws of gravity have an intriguing similarity with the laws of thermodynamics and hydrodynamics - this has been pointed out at several occasions in the physics literature, in particular in connection to the study of black holes (see for example $[8,40,62]$ ). As a consequence one is lead to ask whether gravity can be seen as an emergent effect of an underlying microscopic theory in a thermodynamical limit [40]? The aim of this note is to propose a situation where this question can be answered in affirmatively. We will consider Einstein's vacuum field equations in Euclidean signature on a compact manifold $X$, whose solutions are usually called Einstein metrics in the mathematics literature [5]. More precisely, these equations will be considered in the presence of a fixed background integrable complex structure $J$ on $X$. It turns out that the underlying microscopic theory may then be realized as a certain free fermion gas on $X$ (whose definition only involves $J$ but no metric data) and it will be shown how to recover an Einstein metric (with a non-zero cosmological constant) in the thermodynamical limit. The metric is singled out by the fact that it is Hermitian and Kähler with respect to $J$. In other words these are the Kähler-Einstein metrics which have been extensively studied during the last decade in the mathematics literature (for a recent survey see [52]).

Physically, metrics as above appear, for example, as gravitational instantons in Hawking's functional integral approach to quantum gravity [37, 59]. Although we will not restrict $X$ to be a real four-manifold - the physically most relevant case - it is worth pointing out that in this latter case "most" Einstein metrics are Kähler-Einstein metrics. In particular, for a negative cosmological constant $\Lambda$ it may actually be that all Einstein metrics are Kähler with respect to some complex structure $J$, as long as $X$ complex structures (the question was raised in [47]). This is for example the case for compact quotients of the unit ball, as shown in [46] using Seiberg-Witten gauge theory. By making the complex structure $J$ dynamical, inspired by the works of Fujiki [33] and Donaldson [31] in Kähler geometry, we will also explain how the microscopic theory referred to above can be used to give a finite $N$ well-defined approximation to a variant of quantum gravity (related to Liouville gravity when $n=1$ ) where the role of the diffeomorphism group in ordinary gravity 
is played by a symplectomorphism group which arises as the gauge group for a bundle over the moduli space of all complex structures on the smooth manifold $X$.

To be a bit more precise, given a complex structure $J$ on $X$ we will obtain a canonical (metric free) probability measure $\mu^{(N)}$, expressed in terms of a Slater determinant, on the $N$ - product $X^{N}$ such that in the large $N$-limit the measure $\mu^{(N)}$ becomes exponentially concentrated on configurations of points approximating the normalized volume form $\mu_{K E}$ of the Kähler-Einstein metric of $(X, J)$, i.e. such that

$$
\frac{1}{N} \sum_{i=1}^{N} \delta_{x_{i}} \approx \mu_{K E}
$$

Since the corresponding Kähler-Einstein metric on $(X, J)$ is uniquely determined by its volume form $\mu_{K E}$ this means that the Kähler-Einstein metric indeed emerges macroscopically. Finally, by working over the moduli space of all complex structures on $X$ the dependence on $J$ is also taken into account.

The main ingredients in the investigation of the thermodynamical limit is the asymptotics of exponentially small fluctuations of Slater determinants for $N$-particle correlations of fermions on complex manifolds in [11] (building on [15, 16, 17]). On one hand, from a purely mathematical point of view these large $N$ asymptotics concern large deviations for certain critical determinantal random point processes, which generalize Random Matrix ensembles previously extensively studied. On the other hand, from a physical point of view the result can be seen as an effective bosonization of a free fermion gas (see section 2.2.1), which in the case of a Riemann surface alternatively can be deduced from the exact bosonization results in $[61,3]$. The large deviation result for the Slater determinant is then combined with a basic large deviation result for a non-interacting classical gas going back to Boltzmann's fundamental work on entropy (called Sanov's theorem in the mathematics literature).

It should however be pointed out that the argument in the present note which combines the two mathematically rigorous results referred to above is not completely rigorous. Basically, it involves an interchange of two limits which needs to be mathematically justified. The mathematical details, as well as various extensions, will be investigated elsewhere, but hopefully the heuristic derivation given here is illuminating from a physical point of view as it involves manipulations that are standard in the functional integral approach to quantum field theory.

Incidentally, in the case of a Riemann surface (i.e. the case when the real dimension $D$ of $X$ is two) the situation studied in the present note is closely related to the previous mathematical study of various $2 D$ ensembles (point vortex systems, plasmas, self-gravitating systems, ...) from the point of view of mean field theory; see $[19,43]$ and references therein. In particular, the corresponding thermodynamical limit was studied in $[19,43]$ as a model of 2D turbulence. However, the higher dimensional situation studied in the present work is analytically considerably more complicated as the resulting limiting mean field equations are fully non-linear (see section 2.2). The reason is that the role of the Laplace operator on a Riemann surface is played by the non-linear Monge-Ampère operator for higher dimensional complex manifolds. A different "linear" higher-dimensional generalization of point vortex systems has previously been consider by Kiessling [44], where the role of the Laplace operator is played by the linear Paneitz operator. It involves conformal geometry of spheres rather than the complex (holomorphic) geometry considered here and the thermodynamical limit is a mean field limit of an explicit gas with logarithmic pair interactions. 
It would be interesting to understand the relation between the present note and the ADS/CFT correspondence [1], which relates gravity in the bulk of a manifold to a conformal field theory on its boundary. This is a realization of t'Hooft's holographic principle. Such a principle has recently been put forward by Verlinde in [62] as the basis of an entropic explanation of gravity. As explained in the concluding section 3.3 the emergence of the Kähler-Einstein metric from the present microscopic model can be interpreted as coming from a fermionic maximum entropy principle.

From the mathematical point of view an important motivation for the present work comes from the Yau-Tian-Donaldson program which relates the analytic problem of existence of extremal metrics in a given Kähler class (i.e. Kähler-Einstein metrics in the case of the canonical class) to algebro-geometric stability conditions (notably $K$-stability; see $[26,27,58,52]$ and references therein). For example, the free energy functional derived below turns out to coincide, in the canonical case, with Mabuchi's $K$-energy, which is usually used to define various notions of $K$-stability. Moreover, the thermodynamical convergence towards a KählerEinstein volume form in section 2 is somewhat "dual" to the convergence of canonically balanced metrics conjectured by Donaldson in [28] and proved in [17] (see section 3.1).

As a conclusion one of the mathematical aims of the present paper is to introduce a "thermodynamical formalism" for Kähler-Einstein metrics and more generally for Monge-Ampère equations of mean field type. A rigorous mathematical account of the corresponding variational calculus is given in [14].

Acknowledgment. Thanks to Kurt Johansson for stimulating my interest in general $\beta$-ensembles and to Bengt Nilsson for comments on a draft of the present paper.

1.1. Geometric setup. Let $X$ be a compact Kähler manifold with $\operatorname{dim}_{\mathbb{C}} X=n$. In other words, we are given a real manifold $(X, J)$ of dimension $D=2 n$ equipped with an integrable complex structure $J$ and admitting an Hermitian metric

$$
\omega=\frac{i}{2} h_{i j} d z^{i} \wedge d \bar{z}^{j}
$$

on the complex tangent bundle $T X$, which is closed: $d \omega=0$ (so that $\omega$ is a symplectic form) where $\omega$ is called the Kähler form (metric). Identifying $\omega$ with a Riemannian metric $g$ compatible with $J$, i.e. $g=\omega(\cdot, J \cdot)$ (or locally $g=\operatorname{Re} h$ ) the vacuum Einstein equations, in Euclidean signature, with a cosmological constant read:

$$
\text { Ric } \omega=\Lambda \omega
$$

when $n>1$ and for general $n$ this is the equation for a Kähler-Einstein metric. After a scaling, we may assume that the cosmological constant $\Lambda$ is 0,1 or -1 . In the following we will be mainly concerned with the latter case, i.e. when the solution $\omega$ is a Kähler metric with constant negative Ricci curvature. As shown in the seminal works of Aubin [6] and Yau [64] such a metric $\omega$ exists precisely when the first Chern class $c_{1}\left(K_{X}\right)$ of the canonical line bundle $K_{X}:=\Lambda^{n}\left(T^{*} X\right)$ is positive, which will henceforth be assumed. The Kähler-Einstein metric $\omega$ is then uniquely determined by the complex structure $J$ and we will denote it by $\omega_{K E}$. When $n=1$, i.e. $X$ is a Riemann surface, this amounts to the classical fact that $X$ admits a metric of constant negative curvature precisely when $X$ has genus at least two. This hyperbolic metric is unique in its conformal class (determined by the complex structure $J$ ) 
The starting point of the existence proof of Aubin and Yau is the basic complex geometric fact that the metric $\omega_{K E}$ is uniquely determined by its volume form $\omega_{K E}^{n} / n$ !, that we will normalize to become a probability measure:

$$
\mu_{K E}:=\frac{\omega_{K E}^{n} / n !}{V n !}
$$

In other words the tensor equation 1.1 reduces to a scalar equation (for the density of $\left.\mu_{K E}\right)$ and the Kähler-Einstein metric $\omega_{K E}$ may then be recovered by

$$
\omega_{K E}=\frac{i}{2 \pi} \partial \bar{\partial} \log \mu_{K E}
$$

i.e. as $\frac{i}{2 \pi}$ times the curvature two form of the metric on the canonical line bundle $K_{X}$ defined by $\mu_{K E}$.

The question raised in the introduction may now be reformulated as "Can the probability measure $\mu_{K E}$ be realized as the (macroscopic) expected distribution of particles in a thermodynamical limit of a (microscopic) statistical mechanical system canonically associated to $X$ ? Moreover, the point is to be able to define the microscopic system without specifying any background metric structure so that the Einstein metric and hence (Euclidean) gravity would emerge macroscopically. It turns out that such a statistical mechanical system can indeed be realized by a certain free fermion gas on $X$, as explained below.

1.2. General statistical mechanics formalism. We start by recalling some basic statistical mechanical formalism. Mathematically a (classical) gas of $N$ identical particles (i.e. a random point process with $N$ particles) is described by a symmetric probability measure $\mu^{(N)}$ on the $N$-fold product $X^{N}$ (the $N$-particle configuration space). In local holomorphic coordinates $Z=\left(z_{1}, \ldots, z_{n}\right)$ on the complex manifold $X$ this means that

$$
\mu^{(N)}=\rho^{(N)}\left(Z_{1}, \ldots, Z_{N}\right) d V\left(Z_{1}\right) \wedge \cdots \wedge d V\left(Z_{N}\right)
$$

where $d V\left(Z_{1}\right):=\left(\frac{i}{2}\right)^{n} d z_{1} \wedge d \bar{z}_{1} \wedge \cdots \wedge d z_{n} \wedge d \bar{z}_{n}$ and where the local $N$-point correlation function $\rho^{(N)}$ is invariant under permutations of the $Z_{i}$ :s (note that the fact that we have not written out any dependence on $\bar{Z}_{i}$ does not indicate that the objects are holomorphic!). Pushing forward $\mu^{(N)}$ to $X^{j}$ one then obtains the corresponding $j$-point correlation measures $\mu_{j}^{(N)}$ on $X^{j}$ and their local densities $\rho_{j}^{(N)}$. We will be mainly concerned with the one-point correlation measure $\mu_{1}^{(N)}$ on $X$, i.e.

$$
\mu_{1}^{(N)}:=\int_{X^{N-1}} \mu^{(N)}
$$

In other words, its local density $\rho_{1}^{(N)}(Z)$ represents the probability of finding a particle in the infinitesimal box $d V\left(Z_{1}\right)$. Yet another (trivially) equivalent formulation representation of $\mu_{1}^{(N)}$ can be given:

$$
\mu_{1}^{(N)}=\left\langle\frac{1}{N} \sum_{i} \delta_{x_{i}}\right\rangle,
$$

where the brackets denote the ensemble mean (expectation) of the random variable

$$
\left(x_{1}, \ldots, x_{N}\right) \mapsto \frac{1}{N} \sum_{i} \delta_{x_{i}}
$$


with values in the space $\mathcal{M}_{1}(X)$ of probability measures on $X$. In other words, if $\phi$ denotes a fixed smooth function then

$$
\int_{X} \phi \mu_{1}^{(N)}=\frac{1}{N} \sum_{i}\left\langle\phi\left(x_{i}\right)\right\rangle=\left\langle\phi\left(x_{1}\right)\right\rangle
$$

We will next explain how to define $\mu^{(N)}$ so that the one-point correlation measures converge to the normalized volume form of the Kähler-Einstein metric:

$$
\mu_{1}^{(N)} \rightarrow \mu_{K E}
$$

in the large $N$-limit. More precisely, the convergence will hold in the weak topology on $\mathcal{M}_{1}(X)$, i.e.

$$
\int_{X} \phi \mu_{1}^{(N)} \rightarrow \int \phi \mu_{K E}
$$

for any fixed smooth function $\phi$ on $X$. This convergence can be interpreted as an answer to the question raised above. In fact, the argument will give a much stronger "exponential" convergence which in particular implies the asymptotic factorization of all $j$-point correlation functions (i.e. propagation of chaos holds).

1.3. Line bundles and Slater determinants. To define $\mu^{(N)}$ first recall that we have assumed that the canonical line bundle $K_{X} \rightarrow X$ is positive (i.e. ample in the sense of algebraic geometry). We next recall some basic facts about line bundles (see for example [3, 24] for introductions aimed at physicists). To any holomorphic line bundle $L \rightarrow X$ there is a naturally associated $N$-dimensional complex vector space $H^{0}(X, L)$ consisting of global holomorphic section of $L \rightarrow X$ and the limit we will be interested is when $L$ is replaced by a large tensor power $L^{\otimes k}$. Since $L$ is assumed ample it follows that the dimension $N=N_{k}$ (which will be the number of particles of our gas) grows with $k$ in the following way:

$$
N_{k}:=\operatorname{dim}_{\mathbb{C}} H^{0}\left(X, L^{\otimes k}\right)=V k^{n}+o\left(k^{n}\right)
$$

where the volume $V>0$. In particular,

$$
N\left(=N_{k}\right) \rightarrow \infty \Leftrightarrow k \rightarrow \infty
$$

We will often omit the subscript $k$ in $N_{k}$.

In physics, $H^{0}(X, L)$ usually arises as the quantum ground state space of a single chiral fermion on $X$ coupled to $L[61,3]$, since it may be realized as the zero of a gauged Dirac operator (once metrics/gauge fields are introduce, as explained below). The corresponding $N$-particle space of fermions is then, according to Pauli's exclusion principle, represented by the top exterior power $\Lambda^{N} H^{0}(X, L)$. In other words this is the maximally filled many particle fermion state. As a consequence it is one-dimensional and may, up to scaling, be represented by the $N$-body state

$$
\Psi\left(x_{1}, \ldots x_{N}\right):=\Psi_{1}\left(x_{1}\right) \wedge \cdots \wedge \Psi_{N}\left(x_{N}\right)
$$

expressed in terms of a given base $\left(\Psi_{I}\right)$ in $H^{0}(X, L)$, where $I=1, \ldots, N$. Locally this means that $\Psi$ may be written as a Slater determinant:

$$
\Psi\left(Z_{1}, \ldots, Z_{N}\right)=\operatorname{det}\left(\Psi_{I}\left(Z_{J}\right)\right)
$$

which hence transforms as a holomorphic section of the line bundle $L^{\otimes N}$ over $X^{N}$. 
1.3.1. Introducing metrics. Usually, one equips $L$ with an Hermitian metric $h_{0}$. Taking the squared point-wise norm $\|\Psi(Z)\|^{2}$ with respect to $h_{0}$ of a section $\Psi$ of $L$ hence gives a scalar function on $X$. Let us briefly recall the notion of curvature in this context. The (Chern) curvature form $\Theta$ of $h_{0}$ is the globally well-defined two-form on $X$ locally defined as follows: if $s$ is a local trivializing holomorphic section of $L$, then

$$
\Theta:=-\partial \bar{\partial} \log \left(\|s\|^{2}\right)
$$

Physically, the curvature form $\Theta$ represents a background magnetic two-form of bidegree $(1,1)$ to which the fermions are minimally coupled. More precisely, the holomorphic structure on $L$ together with the Hermitian metric $h_{0}$ determines a reduction of $L$ to a $U(1)$-bundle and a unique unitary connection $A$ on $L$, i.e. a $U(1)$-gauge field such that its field strength $F_{A}=\Theta$ is of type $(1,1)[3,24]$. Let us briefly recall the local meaning of this correspondence. In terms of a local holomorphic trivialization $s$ of $L$ we may write

$$
\Psi(z)=f(z) s
$$

where $f(z)$ is a local holomorphic function, i.e. $\bar{\partial} f=0$. Writing $h_{0}(z):=\|s\|^{2}=$ $e^{-\Phi}$ for a local function $\Phi$ we then have

$$
\|\Psi(Z)\|^{2}=|f(z)|^{2} e^{-\Phi}=\left|f_{\Phi}(z)\right|^{2}
$$

where $f_{\Phi}:=f(z) e^{-\Phi / 2}$ represents $\Psi$ wrt the unitary (but non-holomorphic) local trivialization $e^{\Phi / 2} s$. Note in particular that

$$
\bar{\partial}_{\Phi} f_{\Phi}=0, \quad \bar{\partial}_{\Phi}=\bar{\partial}+\frac{1}{2} \bar{\partial} \Phi
$$

Hence, setting

$$
A=\frac{1}{2}(\bar{\partial} \Phi-\partial \Phi), \quad F_{A}:=d A=\partial \bar{\partial} \Phi
$$

gives a one-form with values in $i \mathbb{R}$ representing the $U(1)$-gauge field (connection) and the equation 1.5 implies that $f_{\Phi}$ is a zero-mode for the Dirac operator $\mathbb{D}_{A}$ expressed in the unitary trivialization (where $\mathbb{D}_{A}$ also depends on a choice of metric on $X$; compare section 2.2.1 below).

The metric $h_{0}$ is positively curved precisely when the real two-form

$$
\omega:=\frac{i}{2 \pi} \Theta\left(=\frac{i}{2 \pi} \partial \bar{\partial} \Phi\right)
$$

is positive definite, i.e. when it defines a Kähler metric on $X$ (and $\Phi$ is sometimes called the local Kähler potential of $\omega$ ). The line bundle $L$ is ample precisely when it admits some positively curved metric. The normalization above ensures that the cohomology class $[\omega]$, which represents the normalized first Chern class $c_{1}(L)$ is an integer class, i.e. it lies in the integer lattice $H^{2}(X, \mathbb{Z})$ of $H^{2}(X, \mathbb{R})$.

The Hermitian metric $h_{0}$ naturally induces metrics on all tensor powers of $L$ etc. Coming back to the Slater determinant above, the point-wise squared norm with respect to the metric $h_{0}$

$$
\left\|\Psi\left(Z_{1}, \ldots, Z_{N}\right)\right\|^{2}
$$

is, from a physical point of view, proportional to the probability of finding (or creating) particles at the point $Z_{1}, \ldots Z_{N}$ on $X$ in the presence of the corresponding background magnetic field. To normalize it we also need to pick an integration measure $\mu_{0}$ (a volume form) on $X$ so that

$$
\left\|\Psi\left(Z_{1}, \ldots, Z_{N}\right)\right\|^{2} / \mathcal{Z}_{N}, \quad \mathcal{Z}_{N}:=\int_{X^{N}}\|\Psi\|^{2} \mu_{0}^{\otimes N}
$$


is a probability density on $X^{N}$. Since, $\Lambda^{N} H^{0}(X, L)$ is one-dimensional the probability density above is, by homogeneity, independent of the choice of base $\left(\Psi_{I}\right)$ in $H^{0}(X, L)$, but, of course, it does depend on the metric $h_{0}$ on $L$ (or more precisely on the background magnetic field $F_{A}$ ) and also on the integration measure $\mu_{0}$ on $X$.

1.4. The canonical background free ensemble. The main point of the present paper is the simple observation that in the particular case when $L$ is the canonical line bundle $K_{X}$ there is no need to specify any metric on $K_{X}$ if one defines a probability measure on $X^{N}$ by

$$
\mu^{(N)}=\left(\Psi_{1} \wedge \bar{\Psi}_{1} \wedge \cdots \wedge \Psi_{N} \wedge \bar{\Psi}_{N}\right)^{1 / k} / \mathcal{Z}_{\mathcal{N}}
$$

To see this first note that it follows from the very definition of $K_{X}$ that $\left(\Psi_{1} \wedge \bar{\Psi}_{1} \wedge\right.$ $\left.\cdots \wedge \Psi_{N} \wedge \bar{\Psi}_{N}\right)^{1 / k}$ transforms as a (degenerate) volume form on $X^{N}$. The points is that if $\Psi$ is a section of $K_{X}\left(:=\Lambda^{n}(T X)\right) \rightarrow X$ then $\Psi \otimes \bar{\Psi}$ gives a well-defined measure on $X$ (i.e. a degenerate volume form), concretely this is a consequence of the local representation $\Psi=f\left(z_{1}, \ldots, z_{N}\right) d z_{1} \wedge \cdots \wedge d z_{N}$. Hence after dividing by

$\mathcal{Z}_{\mathcal{N}}=\int_{X^{N}}\left(\Psi_{1} \wedge \bar{\Psi}_{1} \wedge \cdots \wedge \Psi_{N} \wedge \bar{\Psi}_{N}\right)^{1 / k}=\int_{X^{N}}\left|\operatorname{det}\left(f_{I}\left(Z_{J}\right)\right)\right|^{2 / k} d V\left(Z_{1}\right) \wedge \cdots \wedge d V\left(Z_{N}\right)$

one obtains a probability measure $\mu^{(N)}$ on $X^{N_{k}}$ which is canonically associated to $\left(X, K_{X}^{\otimes k}\right)$, since by homogeneity it is independent of the base $\left(\Psi_{I}\right)$ in $H^{0}\left(X, K_{X}^{\otimes k}\right)$. Note that when $n=1$, i.e. $X$ is a Riemann surface of genus at least two the space $H^{0}\left(X, K_{X}^{\otimes k}\right)$ arises as the space of spin $2 k$ particles $[61,3]$.

1.5. General $\beta$-ensembles. Before turning to the investigation of the thermodynamical convergence towards the Kähler-Einstein volume form $\mu_{K E}$ it should be pointed out that integer powers of Slater determinants have been used before to model the fractional Quantum Hall effect [45]. More generally we note that the previous construction may be generalized by introducing general $k$-dependent powers $\beta_{k}$ in the Slater determinant. To see this we come back to the general setting of an ample line bundle $L \rightarrow X$ and now fix a background metric $h_{0}$ on $L$ and a volume form $\mu_{0}$ on $X$. To this geometric data we associate the probability measure

$$
\mu^{\left(N_{k}\right)}=\|\Psi\|^{\beta_{k}} \mu_{0}^{\otimes N} / \mathcal{Z}_{\mathcal{N}} .
$$

on $X^{N}$ for a fixed choice of parameters $\beta_{k}$. The case of $L=K_{X}$ considered above is obtained by setting $\beta_{k}=2 / k$, fixing any metric $h_{0}$ on $K_{X}$ and then letting $\mu_{0}=1 / h_{0}$, which defines a volume form on $X$. Then it is easy to see that all factors of $h_{0}$ cancel out leading to the previous canonical construction above. Finally, note that if one defines the Hamiltonian

$$
H^{(N)}:=-\log \|\Psi\|
$$

then $\mu^{\left(N_{k}\right)}$ may be represented as a Boltzmann-Gibbs ensemble

$$
\mu^{\left(N_{k}\right)}=e^{-\beta_{k} H^{(N)}} \mu_{0}^{\otimes N} / \mathcal{Z}_{\mathcal{N}},
$$

of a classical system in thermal equilibrium with an external heat bath of temperature $T_{k}=1 / \beta_{k}$. From this point of view $\mathcal{Z}_{\mathcal{N}}$ is the partition function of the system. It depends of the choice of bases $\left(\Psi_{I}\right)$ in $H^{0}\left(X, L^{\otimes k}\right)$ (but $\mu^{\left(N_{k}\right)}$ does not, as explained above). For example, the case when $\beta_{k}=1,2$ or 4 appears in the study of the Random Matrix ensembles associated to the classical groups (see [42] and references therein). In this latter cases $X$ is taken as the Riemann sphere, i.e. the compactification of $\mathbb{C}$ and then the Hilbert space $H^{0}\left(X, L^{\otimes k}\right)$ appears as the corresponding orthogonal polynomials on $\mathbb{C}$. However, as will be explained below we will 
be different in the case when $\beta_{k}$ depends on $k$ and is of the order $1 / k$ which changes the thermodynamical limit, as compared to ordinary Random Matrix Theory.

It is worth emphasizing that the Hamiltonian $H^{(N)}$ above is not a sum of pair interactions (even to the leading order) when $n>1$. This is closely related to the fact that the mean field equations obtained in section 2.2 are fully non-linear (i.e. non-linear in the derivative terms, so that the corresponding actions are of higher order) and it makes the analysis of the thermodynamical limit rather challenging.

\section{Convergence in the thermodynamical Limit}

It will be illuminating to consider the general setting of the previous setting with

$$
\beta_{k}=\beta / k
$$

for a fixed parameter $\beta$ (where $\beta=2$ appears in the canonical background free case 1.4). As will be clear this is, in a certain sense, a mean field limit. As explain above we hence fix the geometric data $\left(h_{0}, \mu_{0}\right)$ consisting of Hermitian metric $h_{0}$ on $L \rightarrow X$ and a volume form $\mu_{0}$ on $X$ (the canonical case is then a special case if one takes $h_{0}=1 / \mu_{0}$ for any given volume form $\left.\mu_{0}\right)$. Given this data we furthermore fix a base $\left(\Psi_{I}\right)$ in $H^{0}\left(X, L^{\otimes k}\right)$, for any $k$, which is orthonormal with respect to Hilbert space structure on $H^{0}\left(X, L^{\otimes k}\right)$ induced by $\left(h_{0}, \mu_{0}\right)$ :

$$
\langle f, g\rangle_{X}:=\int_{X}\langle f, g\rangle \mu_{0}
$$

where the point-wise Hermitian product in the integrand is taken with respect $h_{0}$. In particular, the corresponding partition function is then (a power of) the induced $L^{\beta / k}$ norm of the corresponding Slater determinant $\Psi$ (formula 1.3):

$$
\mathcal{Z}_{N}:=\int_{X^{N}}\|\Psi\|^{\beta / k} \mu_{0}^{\otimes N}
$$

To prove the convergence we will use the techniques of the theory of large deviations. In a nutshell this is a formalism which allows one to give a meaning to the statement that a given sequence of probability measure $\mu^{(N)}$ on $X^{N}$ is "exponentially concentrated on a deterministic macroscopic measure $\mu_{*}$ with a rate functional $I(\mu)$ " (see [60] for an introduction to the theory of large deviations, due to Cramér, Varadhan and others, emphasizing the links to statistical mechanics - relations to functional integrals are emphasized in [35]) . Heuristically, the idea is to think of the large $N$-limit of the $N$-particle space $X^{N}$ of "microstates" as being approximated by a space of "macrostates", which is the space $\mathcal{M}_{1}(X)$ of all probability measures on $X$ :

$$
X^{N} \sim \mathcal{M}_{1}(X)
$$

as $N \rightarrow \infty$. The exponential concentration referred to above may then be heuristically written as

$$
\mu^{(N)}:=\rho^{(N)}\left(Z_{1}, \ldots, Z_{N}\right) d V\left(Z_{1}\right) \wedge \cdots d V\left(Z_{N}\right) \sim e^{-N F(\mu)} \mathcal{D} \mu,
$$

where $\mathcal{D} \mu$ denotes a (formal) probability measure on the infinite dimensional space $\mathcal{M}_{1}(X)$ (more generally, the exponent $N$ could be replaced by a rate $a_{N}$ which is usually a power of $N$ ), where $F(\mu)$ is called the rate functional. Exponential concentration around $\mu_{*}$ appears when $F(\mu) \geq 0$ with $\mu_{*}$ the unique minimizer of $F$. Mathematically, the "change of variables" from $X^{N}$ to $\mathcal{M}_{1}(X)$ is made precise by using the embedding

$$
j_{N}: X^{N} \rightarrow \mathcal{M}_{1}(X), \quad j_{N}\left(x, \ldots, x_{N}\right):=\frac{1}{N} \sum_{i} \delta_{x_{i}}
$$


and then pushing forward $\mu^{(N)}$ to $\mathcal{M}_{1}(X)$ with the map $j_{N}$, giving a probability measure $\left(j_{N}\right)_{*} \mu^{(N)}$ on $\mathcal{M}_{1}(X)$ (i.e. the law of the random variable 1.2). The precise mathematical meaning of 2.1 , in the sense of large deviations, is then that

$$
\lim _{\delta \rightarrow 0} \lim _{N \rightarrow \infty} \frac{1}{N} \log \int_{\mathcal{B}_{\delta}(\mu)}\left(j_{N}\right)_{*} \mu^{(N)}=-F(\mu),
$$

integrating over a small ball $\mathcal{B}_{\delta}(\mu)$ of radius $\delta$ centered at $\mu \in \mathcal{M}_{1}(X)$ (using any metric on $\mathcal{M}_{1}(X)$ which is compatible with the weak topology) and where we have assumed for simplicity that $F$ is continuous (however in our case the definition of the LDP is slightly more complicated as $F$ will only be semi-continuous).

The idea is now to establish the asymptotics 2.1 for a certain free energy functional $F(\mu)$ which is minimized precisely on a measure $\mu_{*}$ which equals the KählerEinstein measure $\mu_{K E}$ in the canonical case introduced in section 1.4. In fact, in this latter case the functional $F(\mu)$ will turn out to be naturally identified with Mabuchi's K-energy, which plays an important role in Kähler geometry (as explained in section 3.1)

To this end we will combine two already established asymptotics, concerning the the case when $\beta_{k}=0$ and $\beta_{k}=2$ respectively. In the first case it is a classical result going back to the work of Boltzmann (called Sanov's theorem in the mathematics literature) that the asymptotics 2.1 hold with $-F(\mu)$ equal to the relative entropy functional $S(\mu)$ :

$$
\mu_{0}^{\otimes N} \sim e^{N S(\mu)} \mathcal{D} \mu
$$

where

$$
S(\mu):=-\int_{X} \log \left(\frac{\mu}{\mu_{0}}\right) \mu(\leq 0)
$$

if $\mu$ has a density with respect to $\mu_{0}$ and otherwise $S(\mu)=-\infty$. This result gives a precise meaning to Boltzmann's notion of entropy as proportional to the logarithmic number (or volume) of microstates corresponding to a given macrostate.

Next, in the case when $\beta_{k}=2$ it was shown very recently in [11] that

$$
e^{-2 H^{(N)}\left(x_{1}, \ldots, x_{N}\right)} \mu_{0}^{\otimes N} \sim e^{-k N E(\mu)} \mathcal{D} \mu
$$

In the present work we are interested in the intermediate asymptotic regime where $\beta_{k}=\beta / k$. Decomposing the corresponding probability measure $\mu^{(N)}$ as

$$
\mu^{(N)}:=\left(e^{-2 H^{(N)}\left(x_{1}, \ldots, x_{N}\right)}\right)^{\beta / 2 k} \mu_{0}^{\otimes N}
$$

or more precisely as

$$
\mu^{(N)}:=\left[\left(e^{-2 H^{(N)}\left(x_{1}, \ldots, x_{N}\right)} \mu_{0}^{\otimes N}\right)^{\beta / 2 k}\right]\left[\cdot\left(\mu_{0}^{\otimes N}\right)^{1-\beta / 2 k}\right]
$$

we can, at least heuristically, combine the asymptotics 2.2 and 2.4 to get

$$
\mu^{(N)} \sim e^{-N \beta\left(E(\mu)-\frac{1}{\beta} S(\mu)\right)} \mathcal{D} \mu
$$

(in order to be mathematically rigorous this heuristic argument needs to be complemented with precise estimates justifying the "interchange" of the large $N$ and small $\delta$-limits)

The convergence of of the one-point correlation measures $\mu_{1}^{(N)}$ towards the minimizer $\mu_{*}$ of the functional

$$
\left.F(\mu):=E(\mu)-\frac{1}{\beta} S(\mu)\right)
$$


can now be shown by standard arguments (given the existence and uniqueness of $\mu_{*}$ which we will deal with in section 2.2). First note that the partition function may be asymptotically calculated as

$$
\mathcal{Z}_{\mathcal{N}} \sim \int_{\mathcal{M}_{1}(X)} \mathcal{D} \mu e^{-\beta N\left(E(\mu)-\frac{1}{\beta} S(\mu)\right)},
$$

giving

$$
-\frac{1}{\beta N} \log \mathcal{Z}_{\mathcal{N}} \rightarrow \inf _{\mu \in \mathcal{M}_{1}(X)}\left(E(\mu)-\frac{1}{\beta} S(\mu)\right.
$$

Next, note that upon performing an overall scaling of the original base $\left(\Psi_{I}\right)$ we may assume that the infimum above vanishes. Now fix a smooth function $\phi$ on $X$ and consider the functional

$$
\mathcal{F}_{N}(u):=-\log \left\langle e^{-\left(\phi\left(x_{1}\right)+\ldots+\phi\left(x_{N}\right)\right)}\right\rangle:=-\log \int_{X^{N}} e^{-\left(\phi\left(x_{1}\right)+\ldots+\phi\left(x_{N}\right)\right)} \mu^{\left(N_{k}\right)}
$$

The following basic general exact variational identity holds

$$
\frac{1}{\beta N} \frac{d \mathcal{F}_{N}(t \phi)}{d t}{ }_{t=0}=\int_{X} \mu_{1}^{\left(N_{k}\right)} \phi
$$

Arguing precisely as above and using the trivial asymptotics

$$
e^{-\left(\phi\left(x_{1}\right)+\ldots+\phi\left(x_{N}\right)\right)} \sim e^{-N \int_{X} \phi \mu}
$$

hence gives

$$
\frac{1}{\beta N} \mathcal{F}_{N}(t \phi) \rightarrow \inf _{\mu \in \mathcal{M}_{1}(X)}\left(E(\mu)-\frac{1}{\beta} S(\mu)+t \int_{X} \phi \mu\right)
$$

Finally, differentiating with respect to $t$ gives

$$
\frac{1}{\beta N} \frac{d \mathcal{F}_{N}(t \phi)}{d t} \rightarrow 0+\int_{X} \mu_{*} \phi
$$

and hence, using 2.6 , we finally get

$$
\mu_{1}^{(N)} \rightarrow \mu_{*}
$$

Next, we will show that the minimizer $\mu_{*}$ can be obtained by solving a mean field type equation which will reduce to the Kähler-Einstein equation in the canonical case. We will start by explaining the notion of pluricomplex energy $E(\mu)$ appearing in the asymptotics 2.4 .

2.1. The pluricomplex energy $E(\mu)$. Assume now that the fixed metric $h_{0}$ on $L$ has positive curvature, i.e. its normalized curvature form is a Kähler form that we denote by $\omega\left(:=\omega_{0}\right)$. Since, $h_{0}$ is uniquely determined up to scaling by its curvature form $\omega$ and since the probability measure $\mu^{(N)}$ is insensitive to scaling of $h_{0}$ we may as well say that the geometric data defining the $\beta_{k^{-}}$ensemble is $\left(\omega_{0}, \mu_{0}\right)$.

Now any Kähler metric which is cohomologous to $\omega$ (i.e. in the class $[\omega]=c_{1}(L)$ ) may by the $\partial \bar{\partial}$-lemma be written as

$$
\omega_{\phi}:=\omega+\frac{i}{\pi} \partial \bar{\partial} \phi
$$

for a smooth function $u$. In this way the space of all Kähler metrics in $c_{1}(L)$ be identified with the space of (global) Kähler potentials

$$
\mathcal{H}_{\omega}(X):=\left\{\phi \in \mathcal{C}^{\infty}(X): \omega_{\phi}>0\right\}
$$

modulo constants (we will usually $\bmod$ out by $\mathbb{R}$ sometimes without mentioning it explicitly). Note that these potentials are globally well-defined and depend on the choice of $\omega$ Geometrically, the space $\mathcal{H}_{\omega}(X)$ may be identified with the space 
$\mathcal{H}(L)$ of all positively curved Hermitian metrics on $L$ and $\omega_{\phi}$ with the (normalized) curvature form of the metric

$$
h_{\phi}:=e^{-2 \phi} h_{0}
$$

on $L$ corresponding to $\phi$ (as follows immediately from formula 1.4), i.e. $\phi:=\Phi-\Phi_{0}$ in terms of local Kähler potentials of $\omega_{\phi}$ and $\omega$ respectively.

Thanks to Yau's solution of the Calabi conjecture one can also associate potentials to volume forms on $X$. Indeed, to any volume form $\mu$ on $X$ (which we will always assume normalized so that $\left.\int_{X} \mu=1\right)$ there is a unique potential $\phi\left(:=\phi_{\mu}\right)$ in $\mathcal{H}_{\omega}(X) / \mathbb{R}$ such that

$$
\frac{\omega_{\phi}^{n}}{V n !}=\mu
$$

where $V$ is the volume of any Kähler metric in the class $c_{1}(L)$. The equation involves the $n$ :th exterior power of $\omega_{\phi}$ and is hence a non-linear generalization of the inhomogeneous Laplace equation, called the inhomogeneous (complex) Monge-Ampère equation (and the left hand side above is called the Monge-Ampère measure of $\phi$ ).

The previous equation can also be given a variational formulation by noting that there is a functional $\mathcal{E}_{\omega}$ (we will often omit the subscript $\omega$ ) on the space $\mathcal{H}_{\omega}(X)$ such that its first variation is given by

$$
\delta \mathcal{E}(\phi):=d \mathcal{E}_{\phi}=\frac{\omega_{\phi}^{n}}{V n !},
$$

where $d \mathcal{E}$ is the differential of $\mathcal{E}(\phi)$ seen as a one-form on $\mathcal{H}_{\omega}(X)$. The functional $\mathcal{E}$ is uniquely determined by the normalization $\mathcal{E}(0)=0$ (singled out by the fixed reference Kähler metric $\omega$ ). This is a well-known functional in Kähler geometry which seems to first have been introduced by Mabuchi ( (it is denoted by $-F_{\omega}$ in the book [58]; similar functionals also appeared in the works of Aubin and Yau). We will call it the Monge-Ampère action, since it physically appears as an action generalizing the Liouville action, as explained in section 2.2.1. It is straight-forward to obtain an explicit formula for $\mathcal{E}(\phi)$ by integrating along the line segment $t \phi$ for $0 \leq t \leq 1$ and get

$$
\mathcal{E}_{\omega}(\phi)=\frac{1}{(n+1) ! V} \int_{X} \phi \sum_{i=1}^{n}\left(\omega^{n-j} \wedge \omega_{\phi}^{j}\right),
$$

but we will only make use of the defining property 2.9 in the following.

The functional $\mathcal{E}$ is (strictly) concave on $\mathcal{H}_{\omega}(X) / \mathbb{R}$ (with respect to the flat metric) [58] and hence the potential $\phi_{\mu}$ may be characterized as the unique (mod $\mathbb{R})$ maximizer of the the functional

$$
\phi \mapsto \mathcal{E}(\phi)-\langle\phi, \mu\rangle,
$$

expressed in terms of the usual pairing

$$
\langle\phi, \mu\rangle:=\int_{X} \phi \mu
$$

Finally, we can now, following [17], define the (pluricomplex) energy $E_{\omega}(\mu)$ of the measure $\mu$ (but we will often omit the subscript $\omega$ ) as

$$
E(\mu):=\sup _{\phi \in \mathcal{H}_{\omega}(X)} \mathcal{E}(\phi)-\langle\phi, \mu\rangle=\mathcal{E}\left(\phi_{\mu}\right)-\left\langle\phi_{\mu}, \mu\right\rangle
$$

The first equality in fact makes sense for any (possibly singular) measure $\mu$ in $\mathcal{M}_{1}(X)$ and one says that $\mu$ has finite energy if $E(\mu)<\infty$.

When $n=1$ one may actually take the sup defining $E$ over all $\phi \in \mathcal{C}^{\infty}(X)$ (i.e. without imposing the constraint $\left.\omega_{\phi}>0\right)$. Then the convex functional $E(\mu)$ is, by definition, the Legendre transform of the concave functional $\mathcal{E}(\phi)$ on $\mathcal{C}^{\infty}(X)$ (with 
a non-standard sign convention). It turns out that in the case $n>1$ the functional $E(\mu)$ can also be realized as a Legendre transform by extending $\mathcal{E}(\phi)$ to another (concave and one time differentiable) functional $\mathcal{F}_{\infty}$ on $\mathcal{C}^{\infty}(X)[17,11,15]$ (which appears in the general asymptotics 2.16 below). This fact is an important ingredient in the variational approach to complex Monge-Ampère equations introduced in [17].

Note that the energy functional $E$ certainly depends on the choice of fixed Kähler metric $\omega$. In fact, it is not hard to see that $E(\mu) \geq 0$ with equality precisely if $\mu=\omega^{n} / V n$ !. Indeed, it follows from general principles (concerning Legendre transforms) that

$$
\inf _{\mu \in \mathcal{M}_{1}(X)} E(\mu)=E((\delta \mathcal{E})(0))=E\left(\frac{\omega^{n}}{V n !}\right)=\mathcal{E}(0)-0=0
$$

It would hence be more appropriate to call $E(\mu)$ the relative energy of $\mu$.

2.1.1. The Riemann surface case. It may be illuminating to consider the case when $n=1$, i.e. when $X$ is a Riemann surface. Then $\mathcal{E}(\phi)$ coincides with the functional sometimes referred to as the Liouville action in the physics literature [3, 51]:

$$
\mathcal{E}(\phi)=\frac{1}{2} \int_{X} \phi\left(\omega_{\phi}+\omega\right)
$$

and hence, taking the potential $\phi_{\mu}$ to be normalized so that $\int \phi_{\mu} \omega=0$ we get

$$
E(\mu)=-\frac{i}{2 \pi} \int_{X} \phi_{\mu} \partial \bar{\partial} \phi_{\mu}=\frac{i}{2 \pi} \int \partial \phi_{\mu} \wedge \bar{\partial} \phi_{\mu}
$$

which is essentially the usual electrostatic energy of the continuous charge distribution $\mu$ in the neutralizing background charge $-\omega$. Equivalently, if we define the Green function $g(x, y)$ for the scalar Laplacian $\Delta=\omega^{-1} \frac{i}{\pi} \partial \bar{\partial}$ on $X$ by the properties $g(x, y)=g(y, x)$ and

$$
\frac{i}{\pi} \partial_{x} \bar{\partial}_{x} g(x, y)=\delta_{x}(y)-\omega(y), \quad \int_{X} g(x, y) \omega(y)=0
$$

then we have $\phi_{\mu}(x)=\int_{X} g(x, y) d \mu(y)$ and hence

$$
E(\mu)=-\frac{1}{2} \int_{X \times X} g(x, y) d \mu(x) \otimes d \mu(y) .
$$

2.2. The minimizer $\mu_{*}$ of the free energy functional $F(\mu)$ and mean field equations. In this section we will give some formal variational arguments to determine the minimizer of $F(\mu)$. See [14] for a rigorous account and further developments. Recall that the free energy functional $F(\mu)$ (for a fixed parameter $\beta>0$ ) on the space $\mathcal{M}_{1}(X)$ of probability measure son $X$ is defined by

$$
F(\mu):=E(\mu)-\frac{1}{\beta} S(\mu)
$$

where $E$ is the energy functional define in the previous section and $S(\mu)$ is the relative entropy 2.3. It follows from basic duality arguments that $F$ is strictly convex on $\mathcal{M}_{1}(X)$ (or rather on the subset where $F$ is finite) and hence admits at most one minimizer. Next we note that $\mu$ is a critical point for $F(\mu)$ on $\mathcal{M}_{1}(X)$ if and only if

$$
-\phi_{\mu}+\frac{1}{\beta} \log \left(\mu / \mu_{0}\right)-Z_{\mu}=0,
$$

where $\phi_{\mu}$ is the potential of $\mu$ and $Z_{\mu}$ is a normalizing constant. Indeed, using the defining properties of $\mathcal{E}$ and $E$ respectively one obtains (by basic Legendre transform considerations) that

$$
\delta E(\mu)=-\phi_{\mu}
$$


as a one-form on the infinite dimensional submanifold $\mathcal{M}_{1}(X)$ of the vector space $\mathcal{M}(X)$ of all signed measures. Moreover, a simple calculation gives

$$
\delta S(\mu)=-\log \left(\mu / \mu_{0}\right)+Z_{\mu},
$$

where $Z_{\mu}$ is a normalizing constant (coming from the constraint $\int_{X} \mu=1$ ). Combining these two variational formulas gives

$$
\delta F(\mu)=-\log \left(\mu / \mu_{0}\right)-\phi_{\mu}
$$

up to a normalizing constant. In other words, $\mu$ is a critical point for for $F(\mu)$ on $\mathcal{M}_{1}(X)$ if and only if its potential $\phi$ solves the following non-linear partial differential equation:

$$
\frac{\omega_{\phi}^{n}}{V n !}=\frac{e^{\beta \phi} \mu_{0}}{Z_{\phi}}
$$

As follows from a simple modification of the proof of the Aubin-Yau theorem there is a unique $\phi \in \mathcal{H}_{\omega}(X) / \mathbb{R}$ solving this equation (crucially using that $\beta>0$ ) which by strict convexity is hence the unique maximizer of the free energy functional $F$. It is sometimes convenient to fix the normalization of the solution $\phi$ above by imposing that

$$
\int_{X} e^{\beta \phi} \mu_{0}=1
$$

i.e. $\phi \in \mathcal{H}_{\omega}(X)$ is the unique solution to

$$
\frac{\omega_{\phi}^{n}}{V n !}=e^{\beta \phi} \mu_{0}
$$

It should be pointed out that when $n=1$ the previous equation is often called the mean field equation [19, 43] and accordingly we will call it the mean field MongeAmpère equation for a general dimension $n$.

Finally, coming back to the canonical case when $\beta=2$ and $L=K_{X}$ we take, as explained in section 1.4 the geometric data $\left(\omega, \mu_{0}\right)$ such that $\omega$ is the curvature form of the metric on $K_{X}$ defined by the inverse $1 / \mu_{0}$. This means that $\mu_{0}=e^{2 f_{\omega}} \omega^{n} / V n$ !, where $f_{\omega}$ is the Ricci potential, i.e.

$$
\frac{i}{\pi} \partial \bar{\partial} f_{\omega}=\omega+\operatorname{Ric} \omega, \int_{X} e^{2 f_{\omega}} \omega^{n} / V n !=1,
$$

where Ric $\omega=-\frac{i}{\pi} \partial \bar{\partial}\left(\log \omega^{n}\right)$ is the $(1,1)$-form representing the Ricci curvature of $\omega$. Then the corresponding Monge-Ampère mean field equation reads

$$
\omega_{\phi}^{n}=e^{2 \phi} e^{2 f_{\omega}} \omega^{n}
$$

Hence, the solution $\phi$ is such that the Kähler metric $\omega_{\phi}$ satisfies

$$
\operatorname{Ric} \omega_{\phi}=-\omega_{\phi},
$$

i.e. $\omega_{\phi}$ is a Kähler-Einstein metric with negative Ricci curvature. Coming back to the convergence of the one-correlation measures in the thermodynamical limit, considered in section 2 , this means that the limiting measure $\mu_{*}$ indeed equals $\mu_{K E}:=\omega_{K E}^{n} / V n !$, as expected.

2.2.1. The asymptotics 2.4 for $\beta_{k}=2$ and effective bosonization. Before continuing let us briefly explain the idea behind the large deviation asymptotics 2.4 proved in [11] and its relation to effective bosonization. The starting point is the basic observation that when $\beta_{k}=2$ the one point correlation function $\rho_{1}^{(N)}$ can be represented as a density of states function:

$$
\rho_{1}^{(N)}(Z)=\sum_{I=1}^{N}\left\|\Psi_{I}(Z)\right\|^{2}
$$


(called the Bergman kernel at the diagonal in the mathematics literature). By a fundamental result of Bouche and Tian the leading asymptotics of the corresponding one point correlation measure are given by the Monge-Ampère measure:

$$
\mu_{1}^{(N)} \rightarrow \omega^{n} / V n !
$$

(see [66] for a survey of Bergman kernel asymptotics and [24] for a physical point of view) Now using these asymptotics and perturbing by potentials $\phi$ in $\mathcal{H}_{\omega}(X)$ one can reverse the arguments used in the end of section 2 and get

$$
\frac{1}{N} \mathcal{F}_{N}(\phi) \rightarrow \mathcal{E}(\phi), \phi \in \mathcal{H}_{\omega}(X)
$$

using the variational property of $\mathcal{E}$. Then an argument involving Legendre transforms gives the large deviation asymptotics 2.4 , using that $E(\mu)$ can be realized as an (infinite dimensional) Legendre transform of $\mathcal{E}(\phi)$. More precisely, the argument uses the convergence of (perturbed) free energies

$$
\frac{1}{N} \mathcal{F}_{N}(\phi) \rightarrow \mathcal{F}_{\infty}(\phi), \phi \in \mathcal{C}^{\infty}(X)
$$

for any smooth function $\phi$ (not necessarily with $\omega_{\phi} \geq 0$ ) for a certain functional $\mathcal{F}_{\infty}$ on $\mathcal{C}^{\infty}(X)$, whose Legendre transform is $E(\mu)$. The key point, as shown in [15], is that $\mathcal{F}_{\infty}$ is one time differentiable on $\mathcal{C}^{\infty}(X)$, which hence establishes the absence of a phase transition with respect to perturbations of $\phi$ for the $\beta_{k}=2$-ensemble.

Incidentally, as explained in [11] the large deviation asymptotics 2.4 can, from a physical point of view, be interpreted as an effective bosonization of a fermionic quantum field theory on $X$ (but is should be pointed out that this is only an interpretation: no bosonization is actually used in the derivation 2.4 as explained above). In other words, the collective theory of $N$ fermions is effectively described by a bosonic field theory, as $N \rightarrow \infty$. The point is that the asymptotics 2.4 is equivalent, at least formally, to the asymptotics

$$
\left\langle\left\|\Psi\left(x_{1}\right)\right\|^{2} \cdots\left\|\Psi\left(x_{N}\right)\right\|^{2}\right\rangle \sim\left\langle e^{i \phi\left(x_{1}\right)} \cdots e^{i \phi\left(x_{N}\right)}\right\rangle,
$$

in the large $N$-limit. Here the lhs above is the usual $N$-point function for a fermionic quantum field theory with the usual gauged Dirac action, i.e. it is the following functional integral over Grassman fields:

$$
\left\langle\left\|\Psi\left(x_{1}\right)\right\|^{2} \cdots\left\|\Psi\left(x_{N}\right)\right\|^{2}\right\rangle:=\int \mathcal{D} \Psi \mathcal{D} \bar{\Psi} e^{-S_{f e r m}(\Psi, \bar{\Psi})}\left\|\Psi\left(x_{1}\right)\right\|^{2} \cdots\left\|\Psi\left(x_{1}\right)\right\|^{2},
$$

integrating of over all complex spinors, i.e. smooth sections of the exterior algebra $\Lambda^{0, *}\left(T^{*} X\right) \otimes L^{\otimes k}$ and where $S_{\text {ferm }}(\Psi, \bar{\Psi})$ is the fermionic action

$$
S_{\text {ferm }}(\Psi, \bar{\Psi})=\int_{X}\left\langle\mathbb{D}_{k A} \Psi, \Psi\right\rangle \mu_{0},
$$

expressed in terms of the gauged Dirac operator $\mathbb{D}_{k A}$ on $\Lambda^{0, *}\left(T^{*} X\right) \otimes L^{\otimes k}$ induced by the complex structure $J$, the Hermitian metric on $L$, i.e. a gauge field $A$ and a choice of Hermitian metric $g$ on $X$ with volume form $\mu_{0}$, i.e. $\mathbb{D}_{k A}=\bar{\partial}+\bar{\partial}^{*}$ a (see [3] for the Riemann surface case). The integer $N$ is the dimension of the space of zero-modes of $\mathbb{D}_{A}$ on $\Lambda^{0, *}\left(T^{*} X\right) \otimes L^{\otimes k}$ which coincides with $H^{0}\left(X, L^{\otimes k}\right)$ due to Kodaira vanishing in positive degrees (when $k>>1$ ) and is hence, in fact, independent of the metric $g$. As for the rhs in 2.17 the bracket denote integration 
wrt the (formal) functional measure $\mathcal{D} \phi e^{-S_{\text {bose }}(\phi)}$ over all scalar field $\phi$ on $X$ and where

$$
S_{\text {bose }}(\phi)=-\frac{1}{(-i)^{n-1}} \mathcal{E}_{-i \omega}(\phi)
$$

defining bosonic action which is a "higher-derivative action" when $n>1$ and when $n=1$ it coincides with non-solotonic part of the bosonic action obtained in $[3,61]$.

\section{Relation to the Yau-Tian-Donaldson program and quantum GRAVITY}

3.1. Duality and relation to the Yau-Tian-Donaldson program and balanced metrics. In this section we will briefly point out some relations to the influential Yau-Tian-Donaldson program in Kähler geometry $[26,27,58,52]$. In a nutshell the idea of this program is to approximate Kähler-Einstein metrics (and more general extremal metrics), by a limit of finite dimensional objects of an algebrogeometrical nature. There are various versions of this program, but the one which is most relevant for the present paper is Donaldson's notion of canonically balanced metrics introduced in [28], which is particularly adapted to Kähler-Einstein metrics (as opposed to general extremal metrics).

To highlight the similarities let us first formulate a more general " $\beta$-analogue" of Donaldson's setting, starting with an ample line bundle $L \rightarrow X$. The main point is to replace the infinite dimensional space $\mathcal{H}_{\omega}(X)$ of Kähler potentials for $\omega \in c_{1}(L)$ (we recall that $\mathcal{H}_{\omega}(X)$ may be identified with the space of all positively curved metrics on $L$ ) with its quantization at level $k$. This latter space, denoted by $\mathcal{H}_{k}$, is the space of all Hermitian metrics on the finite dimensional vector space $H^{0}\left(X, L^{\otimes k}\right)$. Upon fixing a reference metric $\mathcal{H}_{k}$ is hence isomorphic to the symmetric space

$$
G L\left(N_{k}\right) / U\left(N_{k}\right)
$$

of all Hermitian $N_{k} \times N_{k^{-}}$matrices. There is a natural injection defined by the Fubini-Study map $F S_{k}(H)$ at level $k$ :

$$
F S_{k}: \mathcal{H}_{k} \rightarrow \mathcal{H}_{\omega}(X), \quad F S_{k}(H)(x):=\frac{1}{k} \log \sum_{I=1}^{N}\left\|\Psi_{I}(x)\right\|^{2},
$$

expressed in terms of the point-wise norms with respect to the fixed metric $h_{0}^{\otimes k}$ on $L^{\otimes k}$ of a base $\left(\Psi_{I}\right)$ in $H^{0}\left(X ; L^{\otimes k}\right)$ which is orthonormal with respect to $H$. Moreover, for any given $\beta$ we may define a map in the reversed direction that we will call $\mathrm{Hilb}_{k, \beta}$ :

$$
\operatorname{Hilb}_{k, \beta}: \mathcal{H}_{\omega}(X) \rightarrow \mathcal{H}_{k}
$$

defined as follows: $\operatorname{Hilb}_{k, \beta}(\phi)$ is the Hermitian product (or equivalently, Hilbert norm) on $H^{0}\left(X, L^{\otimes k}\right)$ defined by

$$
\langle f, g\rangle_{H i l b_{k, \beta}(\phi)}:=\int_{X}\langle f, g\rangle e^{-k \phi} e^{\beta \phi} \mu_{0}
$$

(note that $\langle\cdot, \cdot\rangle e^{-k \phi}$ is the Hermitian metric on $L^{\otimes k}$ naturally associated to $\phi \in$ $\mathcal{H}_{\omega}(X)$ and the remaining factor $e^{\beta \phi} \mu_{0}$ should be thought of as a specific choice of integration element depending on $\phi$ ). An element $H_{k}$ in $\mathcal{H}_{k}$ will be said to be $\beta$-balanced at level $k$ with respect to $\left(\omega, \mu_{0}\right)$ if is is a fixed point under the composed map

$$
T_{k, \beta}:=\operatorname{Hilb}_{k, \beta}(\phi){ }_{15} F S_{k}: \mathcal{H}_{k} \rightarrow \mathcal{H}_{k}
$$


on $\mathcal{H}_{k}$. Equivalently, this means that $H_{k}$ is a critical point of the following functional $\mathcal{G}_{k}$ on $\mathcal{H}_{k}$ :

$$
\mathcal{G}_{k}(H):=-\frac{1}{k N} \log \operatorname{det}(H)-\frac{1}{\beta} \log \int_{X} e^{\beta F S_{k}(H)} \mu_{0},
$$

(after normalization). Repeating the arguments in the proof of Theorem 7.1 in [17] concerning the canonical case when $L=K_{X}$ ((the case referred to as $S_{+}$in [17]) essentially word for word, one obtains the existence and uniqueness of a $H_{k} \in \mathcal{H}_{k}$ which is $\beta$-balanced at level $k$ with respect to $\left(\omega, \mu_{0}\right)$ and such that

$$
F S_{k}(H) \rightarrow u_{\beta},
$$

in $\mathcal{H}_{\omega}(X)$ when $k \rightarrow \infty$ (or equivalently, $N \rightarrow \infty$ ) where $u_{\beta}$ is the unique solution of the Monge-Ampère mean field equation 2.15, assuming $\beta>0$. As explained in [17] the main point of the proof is to show that any limit point in $\mathcal{H}_{\omega}(X) / \mathbb{R}$ of the sequence $F S_{k}(H)$ is a maximizer of the following functional on $\mathcal{H}_{\omega}(X)$ :

$$
\mathcal{G}(\phi):=\mathcal{E}(\phi)-\frac{1}{\beta} \log \int_{X} e^{\beta \phi} \mu_{0},
$$

whose critical points are precisely the solutions of the Monge-Ampère mean field equation 2.14. Note that the functional $\mathcal{G}$ is invariant under the natural action by $\mathbb{R}, \phi \rightarrow \phi+c$ and hence maximizing the functional

$$
\mathcal{E}(\phi)-\frac{1}{\beta} \int_{X} e^{\beta \phi} \mu_{0}
$$

picks out the maximizers of $\mathcal{G}$ which satisfies the normalization

$$
\int_{X} e^{\beta \phi} \mu_{0}=1
$$

In the Riemann surface case the functional 3.4 with the exponential term is also sometimes referred to as the Liouville action (it appears for example in Polyakov's functional integral quantization of the bosonic string, further developed in [51])

To see the relation to the $\beta$-ensembles introduced in section 1.5 and their thermodynamical limit one should keep in mind the basic linear duality between functions $\phi$ and measures $\mu$ defined by the basic pairing 2.11 In turn, this pairing induces, using the Legendre transform a non-linear duality between convex functionals of $\phi$ on one hand and convex functionals of $\mu$, on the other.

The roles of the spaces $\mathcal{H}_{\omega}(X)$ and $\mathcal{H}_{k}(X)$ are now played by the space $\mathcal{M}_{1}(X)$ and $\mathcal{M}\left(X^{N_{k}}\right)$, respectively, where $\mathcal{M}_{N_{k}}(X)$ denotes the space of all symmetric probability measures on the product $X^{N}$ (i.e. all $N_{k}$-particle random point processes on $X$ ). The analogue of the Fubini-Study map 3.2 is the map

$$
\mathcal{M}\left(X^{N_{k}}\right) \rightarrow \mathcal{M}_{1}(X), \quad \mu_{N} \mapsto\left(\mu_{N}\right)_{1}:=\left\langle\frac{1}{N} \sum_{i} \delta_{x_{i}}\right\rangle,
$$

sending a random point process to its one-point correlation measure. Finally, the role of a $\beta$-balanced metric is now played by the measure $\mu^{\left(N_{k}\right)} \in \mathcal{M}\left(X^{N_{k}}\right)$ defining the $\beta$-ensemble with $N_{k}$ particles, which was expressed as a Boltzmann-Gibbs ensemble with Hamiltonian $H^{\left(N_{k}\right)}$ in formula 1.7. The point is that $\mu^{\left(N_{k}\right)}$ can also be defined by a variational principle. Indeed, by the $N$-particle Gibbs principle for canonical ensembles $\mu_{\left(N_{k}\right)}$ is the unique minimizer of the $N$-particle mean free energy functional on $\mathcal{M}\left(X^{N_{k}}\right)$ :

$$
F^{(N)}\left(\mu_{N}\right)=\frac{1}{N} \int_{X^{N}} \mu_{N} H^{(N)}-\frac{1}{N} S\left(\mu_{N}, \mu_{0}^{\otimes N}\right)
$$

i.e. the difference between mean energy and mean entropy. There is also an analogue of the definition of a balanced metric as a fixed point of the map $T_{k, \beta}$ above. 
Indeed, it is well-known that any Gibbs-Boltzmann measure can be uniquely determined as a stationary state for a stochastic process $\mu_{t}$ on $X^{N}$ defined by suitable Glauber (or Langevin) dynamics, but we will not develop this point of view here.

Interestingly, performing a Legendre transform of each of the two convex functionals on $\mathcal{M}_{1}(X)$ summing up to the free energy functional $F(\mu)$ (i.e. the pluricomplex energy $E(\mu)$ and minus entropy $-\frac{1}{\beta} S(\mu)$ ) yields a functional on $\mathcal{H}_{\omega}(X)$ which is nothing but the functional $\mathcal{G}$ above:

$$
F=E+\left(-\frac{1}{\beta} S\right), \quad \mathcal{G}=E^{*}+\left(-\frac{1}{\beta} S\right)^{*}
$$

It should be pointed out that in the canonical case (where the critical points of the functionals are Kähler-Einstein metrics) the two functionals $F$ and $\mathcal{G}$ have already appeared in Kähler geometry from a different point of view. For example, the limiting free energy functional $F(\mu)$ on $\mathcal{M}_{1}(X)$ may be identified with Mabuchi's $K$-energy $\nu$ of a Kähler metric in $c_{1}\left(K_{X}\right)$ :

$$
F\left(\omega_{\phi}^{n} / V n !\right)=\nu\left(\omega_{\phi}\right)
$$

The functional $\nu$ was first introduced by Mabuchi as the functional on $\mathcal{H}_{\omega}(X)$ whose gradient with respect to the Mabuchi-Semmes-Donaldson Riemannian metric on $\mathcal{H}_{\omega}(X)$ is the scalar curvature minus its average [58,52]. But this is easily seen to be equivalent to the variational property 2.13 of $F$ and hence $F$ and $\nu$ coincide under the identification above (up to an additive constant). The explicit formula for $\nu$ obtained from the identification 3.6 is in fact equivalent to an explicit formula for $\nu$ of Tian and Chen [58]. Moreover, the functional $-G$ coincides with the Ding called functional (see the book [58] and references therein). Interestingly, using Legendre transforms as above one arrives at new proofs and generalizations of various useful results in Kähler geometry [14].

Finally, it seems worth pointing out that in the case when $\beta=0$ the notion of balanced metrics still makes sense and was studied by Donaldson in [28] with a particular emphasize on the case when $X$ is a Calabi-Yau form. Then $\mu$ may be canonically chosen as $i^{n^{2}} \Omega \wedge \bar{\Omega} / \int i^{n^{2}} \Omega \wedge \bar{\Omega}$ where $\Omega$ is non-vanishing holomorphic $n$-form on $X$ and the curvature forms of the balanced metrics at level $k$ then converge to the unique Ricci flat metric in $[\omega]$, whose existence was established in Yau's proof of the Calabi conjecture. Relation between these balanced metrics on Calabi-Yau manifolds and black holes were considered in [25]. However, in the case when $\beta=0$ the $\beta$-ensembles introduced in the present work appear to be less interesting: they are pure Poisson processes without any connections to fermions. The considerably more complicated case when $\beta$ is negative is briefly discussed below in connection to Kähler-Einstein metrics with positive Ricci curvature.

\subsection{Relations to quantum gravity and the space of all complex struc-}

tures. In Hawking's approach to Euclidean quantum gravity [37] one considers the (formal) functional measure

$$
\mathcal{D} g e^{-S(g)}
$$

on the space of all Riemannian metrics $g$ (modulo diffeomorphisms), where $S(g)$ is the Einstein-Hilbert action with a cosmological constant $\Lambda$ and where we have set the fundamental constants to be equal to one (but eventually we will discuss semi-classical limits). One also has to integrate over all diffeomorphism types of four-manifolds, but the relation to the present setting appears more closely when one considers the restriction of the integration to metrics on a fixed compact smooth manifold $X$. As discussed by Hawking, by choosing a conformal gauge, the integration may be decomposed as an integration over a conformal factor and the space 
of conformal equivalence classes of metrics (see section 5 in [37] and also the very recent paper [38] of t' Hooft ).

To see the relation to the present setting we let $X$ be a smooth oriented manifold admitting some complex structure $J_{0}$ such that the corresponding canonical line bundle $K_{X_{0}}$ is ample (when $n=1$ this just means that $X$ has genus at least two). For simplicity we will also assume that $H^{1}(X, \mathbb{R})=0$. We let $\mathcal{J}(X)$ be the space of all complex structures $J$ on $X$, which are compatible with the orientation. Given $J$ we denote by $\mathcal{K}(X, J)$ be the space of all Kähler metrics on $(X, J)$ in the cohomology class $c_{1}\left(K_{(X, J)}\right)$ and we let $\mathcal{K}(X)$ be their union over all $J$ in $\mathcal{J}(X)$. The group $\operatorname{DIFF}(X)$ of diffeomorphisms on $X$ acts naturally on $\mathcal{J}(X)$ and $K(X)$ and we let $\mathcal{T}(X)$ be the corresponding moduli space $\mathcal{T}(X)=\mathcal{J}(X) / D I F F(X){ }^{1}$ and consider the associated bundle

$$
\mathcal{K}(X) / \operatorname{DIFF}(X) \rightarrow \mathcal{T}(X)
$$

of equivalence classes of Kähler metrics. This bundle may be given a useful complex geometric realization as follows. First we recall that there is a natural holomorphic fibration (the universal family)

$$
\mathcal{X} \rightarrow \mathcal{T}(X)
$$

such the fiber $\mathcal{X}_{[J]}$ is a complex manifold biholomorphic to $(X, J)$ (when $n=1 \mathcal{X}$ is called the universal curve over $\mathcal{T}(X))$. The bundle 3.7 may now be identified with an infinite dimensional bundle of metrics over $\mathcal{T}(X)$ such that the fiber over $[J]$ gets identified with the corresponding space of Kähler metrics on $\mathcal{X}_{[J]}$. Equivalently,

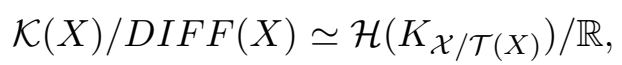

where the rhs is the space of all fiber-wise positively curved metrics on the relative canonical line bundle $K_{\mathcal{X} / \mathcal{T}(X)}$ over $\mathcal{X}$ :

$$
K_{\mathcal{X} / \mathcal{T}(X)} \rightarrow \mathcal{X} \rightarrow \mathcal{T}(X)
$$

(by definition the restriction of $K_{\mathcal{X} / \mathcal{T}(X)}$ to each fiber is the usual canonical line bundle). We can now consider a (formal) functional measure on the space $\mathcal{K}(X) / \operatorname{DIFF}(X)$ of the form

$$
\mathcal{D} g e^{-\frac{1}{N} F(g)}
$$

where the positive integer $N$ plays the role of a semi-classical parameter and we simply let

$$
F(g):=F\left(\mu_{g}\right):=F\left(\frac{d V o l_{g}}{V}\right)
$$

where $F(\mu)$ is the free energy functional defined in section 2 and where we have used that $g$ may be identified with a Kähler metric $\omega_{g}$ in $c_{1}\left(K_{\mathcal{X}_{[J]}}\right)$ for some $J$, so that $d V o l_{g}=\omega_{g}^{n} / n$ !. In other words, fiber-wise over $\mathcal{T}(X)$ the functional $F(g)$ may be identified with the free energy functional for a fixed complex manifold dimorphic to $X$ (which in turn as explained in the previous section may be identified with Mabuchi's $K$-energy). Using the previous arguments we can now give a welldefined and canonical "finite $N$ approximation" to the functional measure 3.8 as follows. First note that for each fiber $\mathcal{X}_{[J]}$ we have the canonical probability measure $\mu^{(N)}$ on $\mathcal{X}_{[J]}^{N}$ (defined in terms of fermions in section 1.4). Moreover the base $\mathcal{T}(X)$ admits a canonical metric, the Weil-Peterson metric, with finite volume (as follows

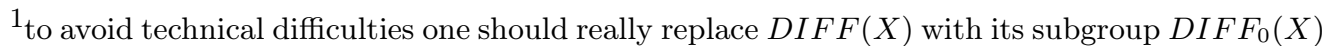
in the connected component of the identity and accordingly the corresponding moduli space with the Teichmuller space associated to $X$ but to simplify the discussion we will not make this distinction. For mathematical results about the relevant moduli spaces we refer the reader to [34, 55].
} 
form the results in [55]). Altogether this induces a "universal" probability measure $\tilde{\mu}^{(N)}$ on the finite-dimensional complex manifold

$$
\mathcal{X}^{(N)}:=\bigcup_{[J] \in \mathcal{T}(X)} \mathcal{X}_{[J]}^{N}
$$

and the arguments in section 2 give the asymptotics

$$
\mathcal{D} g e^{-\frac{1}{N} F(g)} \sim \tilde{\mu}^{(N)}
$$

as $N \rightarrow \infty$ in a suitable sense, so that the measure $\tilde{\mu}^{(N)}$ is exponentially concentrated on the moduli space of all Einstein metrics on $X$ which are Kähler for some complex structure. To see the relation to Hawking's setting one may think of $\mathcal{T}(X)$ as playing the role of the space of all conformal equivalence classes and the Kähler potentials as the logarithm of the conformal factors. This is more then an analogy when $n=1$ since the space $\mathcal{K}(X)$ then coincides with the space of all Riemannian metrics on $X$, normalized so that their total area is 2 genus $(X)-2$. However, in higher dimensions non-trivial integrability conditions appear. Of course, another difference is that the action $F(g)$ is not coming form the Einstein-Hilbert action (in any dimension). As explained in the previous section the action is, in a sense, dual to an action generalizing the Liouville action to higher dimension.

Finally, following Fujiki [33] and Donaldson [31] we will explain how the action $F$ appears naturally from a symplecto-geometric point of view when the complex structures $J$ are used as dynamical variable instead of Riemannian metrics (we refer to $[33,31]$ for more details and further references). Fix a symplectic form $\omega$ in the real cohomology class $\left.-c_{1}(X)=: c_{1}\left(K_{\left(X, J_{0}\right)}\right)\right)$ in $H^{2}(X, \mathbb{Z})$ for any $J \in \mathcal{J}(X)$ and consider the subspace $\mathcal{J}(X, \omega) \subset \mathcal{J}(X)$ of all complex structures $J$ on $X$ which are compatible with $\omega$, i.e. such that

$$
\mathcal{J}(X, \omega) \rightarrow \mathcal{G}(X, \omega): \quad J \mapsto g_{(\omega, J)}:=\omega(\cdot, J \cdot)
$$

maps $J$ to a Riemannian metric which is Kähler wrt $J$ and with $\omega$ as its Kähler form. The map 3.9 naturally defines a principal $\operatorname{SDIFF}(X, \omega)$-bundle over $\mathcal{G}(X, \omega)$, where $\operatorname{SDIFF}(X, \omega)$ is the symplectomorphism group, i.e. $\operatorname{SDIFF}(X, \omega)$ acts freely by pull-back on the fibers so that

$$
\mathcal{J}(X, \omega) / \operatorname{SDIFF}(X, \omega) \simeq \mathcal{G}(X, \omega)
$$

The infinite dimensional space $\mathcal{J}(X, \omega)$ is itself naturally a Kähler manifold with a Weil-Petersson type Kähler form $\Omega$ such that $\operatorname{SDIFF}(X, \omega)$ acts by holomorphic symplectomorphism on $(\mathcal{J}(X, \omega), \Omega)$. More precisely, the action lifts to an Hermitian line bundle over $\mathcal{J}(X, \omega)$ with curvature $\Omega$ can be realized as a certain determinant line bundle (or alternatively as a Deligne pairing [52]). This allows one to apply the powerful formalism of "moment maps" for group actions on Kähler manifolds and geometric invariant theory (in this case the corresponding moment map turns out to be represented by the scalar curvature). Even though the complexified group $\operatorname{SDIFF}(X, \omega) \mathbb{C}$ does not exist, in the strict sense, it is still possible to give a meaning to its orbits in $\mathcal{J}(X, \omega)$ which are complex submanifolds of $\mathcal{J}(X, \omega)$. On each orbit the Kähler metric $\Omega$ has an $\operatorname{SDIFF}(X, \omega)$ - invariant Kähler potential $F(J)$ which is defined up to an additive constant (corresponding to the "norm-functional" / "Kempf-Ness functional" in the abstract setting of moment maps). The functional $F(J)$ may be uniquely determined by requiring its infimum to be equal to 0 (in general the "norm functional" is bounded from below precisely when the group action is semi-stable which is indeed the case in our setting). Finally, for any fixed complex structure $J_{0}$ the quotient of the orbit of $\operatorname{SDIFF}(X, \omega)_{\mathbb{C}}$ passing through $J_{0}$, i.e.

$$
\left(\operatorname{SDIFF}(X, \omega)_{\mathbb{C}} J_{0}\right) / \operatorname{SDIFF}(X, \omega)
$$


may, under the map 3.9, be identified with the space of all Kähler metrics in $c_{1}\left(K_{\left(X, J_{0}\right)}\right)$ (this is the $N=\infty$ analogue of 3.1). Under this identification $F(J)$ coincides with the corresponding free energy functional (since, as shown by Fujiki and Donaldson it coincides with Mabuchi's $K$-energy functional on the space of Kähler metrics). Concretely, the identification used above is obtained by noting that $J$ is in the orbit $\operatorname{SDIFF}(X, \omega)_{\mathbb{C}} J_{0}$ iff there is $f \in D I F F(X)$ such that the pull-back $f^{*} \omega$ defines a Kähler form on $\left(X, J_{0}\right)$ which is cohomologous to $\omega$.

In conclusion this means that from one point of view the space $K(X) / D I F F(X)$ where the functional measure 3.8 lives may be identified with the space of positively curved metrics on the relative canonical line bundle $K_{\mathcal{X} / \mathcal{T}(X)}(\bmod \mathbb{R})$ and the action $F(g)$ with the free energy functional, fiber-wise over the moduli space $\mathcal{T}(X)$. From another point of view $K(X) / D I F F(X)$ may be identified with $\mathcal{J}(X, \omega) / \operatorname{SDIFF}(X, \omega)$ and the corresponding action $F(g)$ with a (normalized) $S D I F F(X, \omega)$-invariant Kähler potential $F(J)$ on the infinite dimensional Kähler manifold $\mathcal{J}(X, \omega)$. In other words, when formulating the theory directly on the space $\mathcal{J}(X, \omega)$ it becomes a gauge theory with structure group $\operatorname{SDIFF}(X, \omega)$ for a bundle over the moduli space of complex structures. The finite $N$-approximation is obtained by replacing $K(X) / D I F F(X)$ with the finite dimensional complex manifold $\mathcal{X}^{(N)}$ fibered over $\mathcal{T}(X)$ and replacing the formal functional measure with a probability measure on $\mathcal{X}^{(N)}$. Finally we note that another somewhat dual approximation, in the spirit of the Yau-Tian- Donaldson program discussed in the previous section, could also be obtained by instead replacing $\mathcal{K}(X) / D I F F(X)$ with the space of Hermitian metrics on the vector bundle $E_{N} \rightarrow \mathcal{T}(X)$ defined as the the direct image of $K_{\mathcal{X} / \mathcal{T}(X)}^{\otimes k}$, i.e.

$$
E_{N_{k}}:=\bigcup_{[J] \in \mathcal{T}(X)} H^{0}\left(\mathcal{X}_{[J]}, K_{\mathcal{X}_{[J]}}^{\otimes k}\right)
$$

(compare the setup in [12]). Note that in this latter case the structure group is $U(N)$ and, in a sense, the Yau-Tian- Donaldson program concerns the problem of making the heuristic statement $U(N) \rightarrow \operatorname{SDIFF}(X, \omega)$, as $N \rightarrow \infty$, mathematically precise.

3.3. Conclusion and outlook. To a given a compact manifold with a fixed integrable complex structure $J$ we have associated a canonical $N$-particle free fermion gas whose one-particle correlation measures converge in the thermodynamical (large $N)$ limit to the volume form of the Kähler-Einstein metric $\omega_{K E}$ associated to $(X, J)$. More precisely, it was assumed that the canonical line bundle $K_{X}$ be positive (i.e. ample), which corresponds to $\omega_{K E}$ having negative Ricci curvature and the oneparticle quantum state space of the fermion gas was taken as the $N_{k}$-dimensional space $H^{0}\left(X, K_{X}^{\otimes k}\right)$ of global holomorphic sections on $X$ with values in $K_{X}^{\otimes k}$. The argument in fact gave precise exponentially small fluctuations around the KählerEinstein volume forms with a rate function $F$ naturally identified with Mabuchi's $K$-energy (which plays an important rule in Kähler geometry). The convergence in the thermodynamical limit was obtained by introducing an auxiliary background Kähler form $\omega$ in the first Chern class $c_{1}\left(K_{X}\right)$ (or equivalently a metric on $K_{X}$ ) also determining a volume form on $X$. This lead to a decomposition of the rate functional $F$ as

$$
F(\mu)=E(\mu)-S(\mu)
$$

(with both terms depending on the choice of $\omega$ ). In terms of the statistical mechanics of a classical canonical Boltzmann-Gibbs ensemble $E$ and $S$ appeared as the limiting mean energy and mean entropy, respectively. Minimizing $F$ then gave an equation of mean field type whose unique solution is given by the Kähler-Einstein volume 
form. An interpretation of the energy $E$ in terms of effective bosonization was also given.

Heuristically, it seems that one could interpret the thermodynamical limit above as saying that the Kähler-Einstein metric emerges from a fermionic maximum entropy principle: the particles try to maximize their entropy (i.e. volume in configuration space) under the constraint that they behave as fermions and hence, according to Pauli's exclusion principle, cannot occupy the same space leading to an effective repulsion.

By making the complex structure $J$ dynamical relations to a variant of quantum gravity were explained using a canonical finite $N$ approximation of the functional measure. It would be interesting to compare with other discretization schemes such as spin foam loop quantum gravity or the approach of CDT (causal dynamical triangulations); see $[50,48,4]$ and references therein. However, in the present case one only consider fluctuating Riemannian metrics which are Kähler with respect to some complex structure on $X$ (imposing a constraint unless $n=1$ ).

The precise mathematical details of the thermodynamical convergence will be investigated elsewhere, as well as the case when the anti-canonica line bundle $K_{X}^{-1}$ is positive (so that any Kähler-Einstein metric must have positive Ricci curvature). In the latter case there are well-known obstructions to the existence of Kähler-Einstein metrics and the Yau-Tian-Donaldson program aims at showing that all obstruction may be formulated in terms of algebro-geometric stability. More precisely, according to the the Yau-Tian-Donaldson conjecture the existence of Kähler-Einstein is equivalent $X$ beeing $K$-stable (or to some refined notion of $K$-stability) [52]. From the point of view of the present paper this is related to the fact that the natural candidate for the $N$-particle ensemble in the case when $K_{X}^{-1}$ is positive may be formally written as

$$
\mu^{(N)}=\left(\Psi_{1}\left(x_{1}\right) \wedge \bar{\Psi}_{1}\left(x_{1}\right) \wedge \cdots \wedge \Psi_{N}\left(x_{N}\right) \wedge \bar{\Psi}_{N}\left(x_{N}\right)\right)^{-1 / k} / \mathcal{Z}_{N}
$$

where now $\left(\Psi_{I}\right)$ is a base in $H^{0}\left(X,\left(K_{X}^{-1}\right)^{\otimes k}\right)$. However, because of the negative exponent above this singular volume form on $X^{N}$ may be non-integrable, i.e. $\mathcal{Z}_{N}=$ $\infty$, due to singularities appearing when to points merge. This means that the correponding Boltszmann-Gibbs measure $\mu^{\left(N_{k}\right)}$ at level $k$ may not even exist. In fact, its existence should be closely relatated to the existence of balanced metrics, which in turn is closely related to the notion of $K$-stability (compare the disucssion in section 3.1). Anyway, fixing an auxiliary smooth Hermitian metric $h=e^{-\Phi_{0}}$ on $K_{X}^{-1}$ one can look at the $\beta$-ensemble

$$
\mu_{\beta}^{(N)}=\left|\Psi_{1} \wedge \bar{\Psi}_{1} \wedge \cdots \wedge \Psi_{N}\right|^{\beta / k}\left(\left(e^{\Phi}\right)^{\otimes N}\right)^{(-2-\beta)} / \mathcal{Z}_{\beta, N}
$$

(with $\beta=-\gamma$ negative) which is integrable for $\gamma$ sufficiently small, coinciding with 3.11 for $\beta=2$. In fact, it can be shown that the measure is integrable as long as $\gamma<\alpha_{X}$, where $\alpha_{X}$ is Tian's $\alpha$-invariant [58] (also called the log canonical threshold in algebraic geometry ). Repeating the same argument as before the rate functional in 2.5 may be written as

$$
\beta F_{\beta}(\mu)=\beta E(\mu)-S(\mu)
$$

In the case of main interest, i.e. $\beta=-2$, this functional may be identified with Mabuchi's $K$-energy functional in Kähler geometry. Since now $\beta<0$ this means that the energy contribution $\beta E(\mu)$ corresponds to an attractive force. Hence, minimizimgs $\beta F_{\beta}(\mu)$ amounts to finding a balance between minimizing energy (which brings the particles close together) and maximizing entropy (which rather has a repelling effect). Accordingly, if $\beta$ is too small then the attractive effect wins and the rate funcional 3.13 becomes unbounded from below. Interestingly, it can be 
shown that when $X$ admits a Kähler-Einstein metrics with volume form $\mu_{K E}$ and holomorphic vector fields (so that the metric is non-unique) then the rate functional 3.13 is bounded from below for $\beta \geq-2$ (with minimizer $\mu_{K E}$ for $\beta=-2$ ) and it is unbounded from below when $\beta<-2$. In fact, the notion of analytic $K$-stability which by Tian's theorem is equivalent to the existence of a unique Kähler-Einstein metric [58] may be equivalently formulated as the condition that $\beta F_{\beta}$ be bounded from below for all $\beta$ sufficently close to -2 (see [14] and references therein). As suggested above it seems natural to expect that the ordinary notion of (non-analytic) $K$-stability is closely related to integrability of the measure 3.11 for $N$ sufficently large. In turn, by Gibbs principle this latter property is equivalent to the boundedness from below of the functional $\beta F^{(N)}$, where $F^{(N)}$ is the corresponding $N$-particle Gibbs free energy (formula 3.5).

If $(X, J)$ admits a Kähler-Einstein metric (or if appropriate stability conditions hold) it seems natural to expect the one-point correlation measure of $\mu_{\beta}^{(N)}$ to converge to a Kähler-Einstein volume form when first $N \rightarrow \infty$ and then $\beta \rightarrow-2$. The simplest case appears when $X$ is the Riemann sphere. Then the corresponding ensemble is explicitly given by a one component plasma (or equivalently a point vortex system) studied by Kiessling in [44] where a first order phase transition appears at $\beta=-2$. Kiessling also considered higherdimensional generalizations in other directions than the one explored here, namely to the conformal geometry of higher dimensional spheres where the correponding Hamiltonian $H^{(N)}$ is a sum of logarithmic pair interaction. As a consequence the corresponding mean field equations are quasi-linear (with the non-linearity coming from the exponential term), as opposed to the present setting where the fully non-linear Monge-Ampère operator appears.

There is also an interesting variant of the probability measure $\mu_{\beta}^{(N)}$ above where one, in the definition 3.12, instead uses a singular metric on $K_{X}^{-1}$ whose curvature is the current $\delta_{D}$ defined by a given Calabi-Yau submanifold $D$ of dimenion $n-1$ in $X$ (such $D$ usually exist); more precisely, $D$ is cut out by a holomorphic section $s$ of $K_{X}^{-1}$ and one takes $\Phi=\log |s|^{2}$ in 3.12. Then the minimizers $\mu_{\gamma}\left(=\omega_{\gamma}^{n} / n\right)$ of the corresponding scaled free energy functional $\beta F_{\beta, D}(\mu)$ (now writing $\beta=-2 \gamma$ for $0<\gamma<1)$ turn out to satisfy an equation very recently introduced by Donaldson [29] (see also [30]):

$$
\operatorname{Ric} \omega_{\gamma}=\gamma \omega_{\gamma}+(1-\gamma) \delta_{D}
$$

saying that $\omega_{\gamma}$ is a Kähler-Einstein metric on $X-D$ whose Ricci curvature is singular along the submanifold (divisor) $D$. The equationa above appeared in a program proposed by Donaldson to attack the Yau-Tian-Donaldson conjecture where the first step consists in proving the existence of solutions to the previous equation for $\gamma<<1$ and the last step amounts to, assumping $K$-stabilility (or rather a variant like $\bar{K}$-stability), use certain balanced metrics to take the limit $\gamma \rightarrow 1$ (corresponding to $\beta \rightarrow-2$ in the present notation) to obtain a bona fida KählerEinstein metric on $X{ }^{2}$

In the light of Donaldson's program it seems natural to formulate the following conjecture: if $X$ is $K$-stable (or say $\bar{K}$-stable in Donaldson's sense) and $X$ contains a submanifold $D$ as above, then the corresponding probability measures $\mu_{\beta, D}^{(N)}$ are

\footnotetext{
${ }^{2}$ The existence of the metric in $3.14 \omega_{\gamma}$ for $\gamma<<1$ has now been proved in [14] using a variational calculus inspired by (but independent of) the statistical mechanical model proposed in the present paper. Moreover, this result combined with the very recent paper [41] shows that $\omega_{\gamma}$ in fact has conical singularies with angle $2 \pi \gamma$ transversally to $D$ and is smooth in the directions of $D$ as also conjectured by Donaldson.
} 
well-defined for all $\beta<-2$ and the corresponding one-point correlation measures converge to a Kähler-Einstein volume form when first $N \rightarrow \infty$ and then $\beta \rightarrow-2$.

Finally, it would also be interesting to detail the Glauber (Langevin) stochastic dynamics alluded to section 3.1 and investigate a suitable "hydrodynamical" scaling limit (see for example the recent paper [23] for relations between Langevin dynamics and field theories with holomorphic factorization and supersymmetry). This should lead to a deterministic heat-equation type flow on the space of all (smooth) probability measures $\mathcal{M}_{1}(X)$, converging towards the Kähler-Einstein volume form. In fact, a "dual" (in the sense of the previous section) scaling limit of Donaldson's iteration of the map $T_{k, \beta} 3.3$ was shown to converge to the Kähler-Ricci flow in [12].

\section{REFERENCES}

[1] Aharony, O; Gubser, SS; Maldacena, J; Ooguri, H; Oz, Y: Large N field theories, string theory and gravity. Physics Reports, 2000 - Elsevier

[2] Aliev, A N; Nutku, N: Gravitational instantons admit hyper-Kähler structure. Class. Quantum Grav. 16 (1999) 189-210.

[3] Alvarez-Gaumé, L; Bost, J-B; Moore, G; Nelson, P; Vafa, C: Bosonization on higher genus Riemann surfaces. Comm. Math. Phys. 112 (1987), no. 3,

[4] Ambjorn, J; Goerlich, A: Jurkiewicz, J; R. Loll: CDT-an Entropic Theory of Quantum Gravity. arXiv:1007.2560

[5] Andersson, M T: A survey of Einstein metrics on 4-manifolds, in: Handbook of Geometric Analysis, in Honor of S.-T. Yau, Ed. Lizhen Ji, R. Schoen et al, International Press, Boston, (2008).

[6] Aubin, T.: Equations du type Monge-Amp'ere sur les vari'et'es k“ahl'eriennes compactes, Bull. Sci. Math. 102 :1 (1978) 63-95

[7] Bando, S.; Mabuchi, T: Uniqueness of Einstein K“"ahler metrics modulo connected group actions, in Algebraic geometry, Sendai, 1985 (T. Oda, Ed.), Adv. Stud. Pure Math. 10, Kinokuniya, 1987, 11-40.

[8] Bardeen, J.M; Carter, B; Hawking, S. W: The four laws of black hole mechanics. Comm. Math. Phys. Volume 31, Number 2 (1973), 161-170.

[9] Ben Arous, G.; Zeitouni, O. Large deviations from the circular law. ESAIM Probab. Statist. 2 (1998), 123-134

[10] Berline, N; Getzler, E; Vergne, M: Heat kernels and Dirac operators. Corrected reprint of the 1992 original. Grundlehren Text Editions. Springer-Verlag, Berlin, 2004.

[11] Berman, R:J: determinantal point processes and fermions on complex manifolds: large deviations and bosonization. Arxiv :0812.4224

[12] Berman, R:J: Relative Kahler-Ricci flows and their quantization. arXiv:1002.3717

[13] Berman, R:J: Analytic torsion, vortices and positive Ricci curvature. arXiv:1006.2988

[14] Berman, R:J: A thermodynamical formalism for Monge-Ampere equations, Moser-Trudinger inequalities and Kahler-Einstein metrics. arXiv:1011.3976

[15] Berman, R.J.; Boucksom, S: Growth of balls of holomorphic sections and energy at equilibrium. Inventiones Mathematicae Vol. 181, Issue 2 (2010), p. 337

[16] Berman, R.J.; Boucksom, S; Witt Nyström, D: Fekete points and convergence towards equilibrium measures on complex manifolds. Acta Mathematica. To appear (arXiv:0907.2820).

[17] Berman, R.J; Boucksom, S; Guedj,V; Zeriahi: A variational approach to complex MongeAmpere equations. http://arxiv.org/abs/0907.4490

[18] Boucksom, S; Essidieux,P: Guedj,V; Zeriahi: Monge-Ampere equations in big cohomology classes. Preprint.

[19] Caglioti.E; Lions, P-L; Marchioro.C; Pulvirenti.M: A special class of stationary flows for two-dimensional Euler equations: a statistical mechanics description. Communications in Mathematical Physics (1992) Volume 143, Number 3, 501-525

[20] Cheltsov, I; Shramov, C: Log canonical thresholds of smooth Fano threefolds. With an appendix by Jean-Pierre Demailly

[21] Deift, P. A. Orthogonal polynomials and random matrices: a Riemann-Hilbert approach. Courant Lecture Notes in Mathematics, 3. New York University, Courant Institute of Mathematical Sciences, New York; American Mathematical Society, Providence, RI, 1999.

[22] Demailly, J-P: Complex analytic and algebraic geometry. Available at www-fourier.ujfgrenoble.fr $/$ demailly/books.html 
[23] Dijkgraaf, R; Orlando, D; Reffert, S: Relating Field Theories via Stochastic Quantization. Nucl.Phys.B824:365-386,2010

[24] Douglas, M.R; Klevtsov, S: Bergman Kernel from Path Integral. Communications in Mathematical Physics, Vol: 293, Issue: 1, January 2010, Pages: 205-230

[25] Douglas, M.R; Klevtsov, S: Black holes and balanced metrics. arXiv:0811.0367

[26] Donaldson, S. K. Scalar curvature and projective embeddings. I. J. Differential Geom. 59 (2001), no. 3, 479-522.

[27] Donaldson, S. K. Scalar curvature and projective embeddings. II. Q. J. Math. 56 (2005), no. $3,345-356$.

[28] Donaldson, , S. K. : Some numerical results in complex differential geometry. arXiv:math/0512625

[29] Donaldson, S.K.: Discussion of the Kahler-Einstein problem. Notes available at http://www2.imperial.ac.uk/ skdona/KENOTES.PDF

[30] Donaldson, S.K.: Kahler metrics with cone singularities along a divisor. arXiv:1102.1196

[31] Donaldson, , S. K.: Remarks on gauge theory, complex geometry and $\$ 4 \$ 4$-manifold topology. Fields Medallists' lectures, 384-403, World Sci. Ser. 20th Century Math., 5, World Sci. Publ., River Edge, NJ, 1997.

[32] Ellis, R: Entropy, large deviations, and statistical mechanics. Springer, 2005

[33] Fujiki, A: The moduli spaces and Kähler metrics of polarized algebraic varieties. (Japanese) Sūgaku 42 (1990), no. 3, 231-243. (Reviewer: Akira Asada),

[34] Fujiki, A; Schumacher, G: The moduli space of extremal compact Kähler manifolds and generalized Weil-Petersson metrics. Publ. Res. Inst. Math. Sci. 26 (1990), no. 1, 101-183.

[35] Gawedzki: K. Lectures on conformal field theory, available at http://www.math.ias.edu/QFT/faIV, or see pp. (727-805) in P. Deligne, D. Freed, (eds.), Classical Field Theory. Quantum Fields and Strings: a Course for Mathematicians, Vol. I, 2 (Princeton, NJ, 1996/1997) Providence, American Mathematical Society 1999.

[36] Griffiths, P; Harris, J: Principles of algebraic geometry. Wiley Classics Library. John Wiley \& Sons, Inc., New York, 1994.

[37] Hawking, S.W.: Spacetime foam. Nuclear Physics B Volume 144, Issues 2-3, 13 November-20 November 1978, Pages 349-362

[38] 't Hooft, G: Probing the small distance structure of canonical quantum gravity using the conformal group. arXiv:1009.0669

[39] Hough, J. B.; Krishnapur, M.; Peres, Y.l; Virág, B: Determinantal processes and independence. Probab. Surv. 3 (2006), 206-229

[40] Jacobson, T: Thermodynamics of Spacetime: The Einstein Equation of State. Phys. Rev. Lett. 75, 1260-1263 (1995)

[41] Jeffres, T; Mazzeo, R; Rubinstein, Y.A:; Kähler-Einstein metrics with edge singularities. Arxiv 1105.5216

[42] Johansson, K: On fluctuations of eigenvalues of random Hermitian matrices. Duke Math. J. 91 (1998), no. 1, 151-204.

[43] Kiessling M.K.H.: Statistical mechanics of classical particles with logarithmic interactions, Comm. Pure Appl. Math. 46 (1993), 27-56.

[44] Kiessling M.K.H.: Statistical mechanics approach to some problems in conformal geometry. Physica A: Statistical Mechanics and its Applications Volume 279, Issues 1-4, 1 May 2000, Pages 353-368

[45] Laughlin, R.B.: Anomalous Quantum Hall Effect: An Incompressible Quantum Fluid with Fractionally Charged Excitations. Phys. Rev. Lett. 50, 1395-1398 (1983)

[46] LeBrun, C: Einstein Metrics and Mostow Rigidity, Math. Res. Lett. 2 (1995) 1-8

[47] LeBrun, C: Einstein Metrics on Complex Surfaces, in Geometry and Physics, Anderson et al. eds. pp. 167-176, Marcel Dekker, 1997.

[48] Loll, R: Discrete approaches to quantum gravity in four dimensions. arXiv:gr-qc/9805049

[49] Macchi, O: The coincidence approach to stochastic point processes. Advances in Appl. Probability 7 (1975), 83-122.

[50] Nicolai, H; Peeters, K: Loop and spin foam quantum gravity: a brief guide for beginners. Lect.NotesPhys.721:151-184,2007 (arXiv:hep-th/0601129)

[51] Onofri1, E; Virasoro, M.A: On a formulation of Polyakov's string theory with regular classical solutions. Nuclear Physics B Vol. 201, Issue 1, 14 June 1982, Pages 159-175

[52] Phong, D.H; Sturm, J: Lectures on Stability and Constant Scalar Curvature. arXiv:0801.4179

[53] Scardicchio, A; Torquato, S; Zachary, C.E: Point processes in arbitrary dimension from fermionic gases, random matrix theory, and number theory . arXiv:0809.0449

[54] Scardicchio, A; Torquato, S; Zachary, C.E: Statistical properties of determinantal point processes in high-dimensional Euclidean spaces. arXiv:0810.4977 
[55] Schumacher, G: Positivity of relative canonical bundles for families of canonically polarized manifolds, arXiv:0808.3259. Curvature of higher direct images and applications. arXiv:1002.4858

[56] Tian, G; Yau S-T: Kähler-Einstein metrics on complex surfaces with $C_{1}>0$ : Comm. Math. Phys. Volume 112, Number 1 (1987), 175-203.

[57] Tian, G; On Calabi's conjecture for complex surfaces with positive first Chern class. Invent. Math. Vol. 101, Nr. 1 (1990)

[58] Tian, G: Canonical metrics in Kähler geometry. Notes taken by Meike Akveld. Lectures in Mathematics ETH Zürich. Birkhäuser Verlag, Basel, 2000.

[59] Tseytlin, A.A: Two-dimensional Kähler Einstein spaces and gravitational instantons. Physics Letters B Vol. 97, Issues 3-4, 15 December 1980, 391-395

[60] Touchette, H: The large deviation approach to statistical mechanics. Physics Reports 478 (2009) 169

[61] Verlinde, E: Verlinde, H: Chiral bosonization, determinants and the string partition function. Nuclear Physics B Volume 288, 1987, Pages 357-396

[62] Verlinde, E: On the Origin of Gravity and the Laws of Newton. . arXiv:1001.0785

[63] Wentworth, R.A.: Precise constants in bosonization formulas on Riemann surfaces II. arXiv:1008.2914

[64] Yau, S.T: On the Ricci curvature of a compact Kähler manifold and the complex MongeAmpère equation. I. Comm. Pure Appl. Math. 31 (1978), no. 3, 339-41

[65] Zabrodin, A; Matrix models and growth processes: from viscous flows to the quantum Hall effect. Preprint in 2004 at arXiv.org/abs/hep-th/0411437

[66] Zelditch, S: Book review of "Holomorphic Morse inequalities and Bergman kernels Journal" (by Xiaonan Ma and George Marinescu) in Bull. Amer. Math. Soc. 46 (2009), 349-361.

[67] Zeitouni O; Zelditch, S: "Large deviations of empirical zero points measures on Riemann surfaces, I: $g=0$. [Preprint].

Chalmers University of Technology and University of Gothenburg

E-mail address: robertb@chalmers.se 